Mon. Not. R. Astron. Soc. 000,115(2010) Printed 10 April $2018 \quad$ (MN LATEX style file v2.2)

\title{
The Growth of Red Sequence Galaxies in a Cosmological Hydrodynamic Simulation
}

\author{
J. M. Gabor, ${ }^{1,2 \star ~ R . ~ D a v e ́ ~}{ }^{2}$ \\ ${ }^{1}$ CEA-Saclay, IRFU, SAp, F-91191 Gif-sur-Yvette, France \\ ${ }^{2}$ University of Arizona, 933 N. Cherry Ave, Tucson, AZ 85721
}

10 April 2018

\begin{abstract}
We examine the cosmic growth of the red sequence in a cosmological hydrodynamic simulation that includes a heuristic prescription for quenching star formation that yields a realistic passive galaxy population today. In this prescription, halos dominated by hot gas are continually heated to prevent their coronae from fueling new star formation. Hot coronae primarily form in halos above $\sim 10^{12} M_{\odot}$, so that galaxies with stellar masses $\sim 10^{10.5} M_{\odot}$ are the first to be quenched and move onto the red sequence at $z>2$. The red sequence is concurrently populated at low masses by satellite galaxies in large halos that are starved of new fuel, resulting in a dip in passive galaxy number densities around $\sim 10^{10} M_{\odot}$. Stellar mass growth continues for galaxies even after joining the red sequence, primarily through minor mergers with a typical mass ratio $\sim 1: 5$. For the most massive systems, the size growth implied by the distribution of merger mass ratios is typically $\sim 2 \times$ the corresponding mass growth, consistent with observations. This model reproduces mass-density and colour-density trends in the local universe, with essentially no evolution to $z=1$, with the hint that such relations may be washed out by $z \sim 2$. Simulated galaxies are increasingly likely to be red at high masses or high local overdensities. In our model, the presence of surrounding hot gas drives the trends both with mass and environment.
\end{abstract}

Key words: galaxies:evolution - galaxies:formation

\section{INTRODUCTION}

A substantial fraction of galaxies today inhabit a tight locus in colour-magnitude space known as the red sequence. Such galaxies typically have elliptical morphologies, little or no star formation, little cold gas, and live in dense environments. They host the majority of stellar mass in the local universe (Hogg et al. 2002), and therefore are a critical population for understanding the global evolution of galaxies across cosmic time.

Despite a growing wealth of data, the origin of red sequence galaxies is still not fully understood. In the currentlyfavored cold dark matter (CDM) paradigm, massive galaxies should be surrounded by halos of hot gas that can potentially cool and fuel new star formation (White \& Frenk 1991). Yet these galaxies show little cold gas or ongoing star formation. Debate continues as to what physical mechanisms can halt star-formation and keep it halted for much of cosmic time, with recent work favoring feedback from active galactic nu-

* Email:jgabor@as.arizona.edu clei (AGN) as the primary energy source (e.g. Croton et al. 2006; Hopkins et al. 2008).

Deep surveys have begun to place constraints on bright passive galaxies over cosmic timescales, out to $z>$ 2 (e.g. Bell et al. 2004; Faber et al. 2007; Brown et al. 2007; Cool et al. 2008; Kriek et al. 2008; Stutz et al. 2008; Taylor et al. 2009; Brammer et al. 2009; Marchesini et al. 2010; Whitaker et al. 2010), and recent surveys should probe down to stellar masses $\sim 10^{9} M_{\odot}$ at such redshifts (e.g. CANDELS; Grogin et al. 2011; Koekemoer et al. 2011). Current data indicates that the red sequence has grown by $\times 2$ from $z \sim 1$ to 0 , but the most massive galaxies were already in place $7+$ Gyrs ago. Furthermore, high-resolution imaging has revealed that passive galaxies are typically much smaller at $z>1$, by factors $\sim 5$ compared to their present-day descendents (Daddi et al. 2005; van Dokkum et al. 2008, 2010). These data provide strong constraints on passive galaxy formation models, and only recently have such constraints been explored in the context of hierarchical models (Khochfar \& Silk 2006; Fan et al. 2008; Nipoti et al. 2009, 2012; Naab et al. 2009; Oser et al. 2010; Shankar et al. 2010; Oser et al. 2012; Cimatti et al. 2012). 
Numerous physical processes are thought to contribute to the formation and evolution of passive galaxies, and many potential effects have proven difficult to rule out. Gas-rich galaxy mergers that induce a starburst and rapid black hole growth may drive the initial quenching of massive red galaxies (Springel et al. 2005; Hopkins et al. 2006, 2008). Alternatively, hot gaseous coronae that form in halos above $\sim$ $10^{12} M_{\odot}$, supplemented by additional heating from an active galactic nucleus (AGN) or another energy source, may starve galaxies of fuel for star-formation (Birnboim \& Dekel 2003; Kereš et al. 2005; Dekel \& Birnboim 2006; Croton et al. 2006; Cattaneo et al. 2006). Among satellite galaxies additional processes may drive quenching: starvation and/or ram-pressure stripping by the hot intergalactic medium, or glancing interactions between galaxies (Gunn \& Gott 1972; Abadi et al. 1999; Quilis et al. 2000; Larson et al. 1980; Bekki et al. 2002; Richstone 1976; Moore et al. 1998). All these processes may act in combination to form passive galaxies as observed.

Once "red and dead," the evolution of these galaxies may be deceptively complex. Although there is little or no new star formation, the stellar population sheds $\sim 1 / 2$ of its mass via stellar winds (Jungwiert et al. 2001; Bruzual \& Charlot 2003). Mergers can alter galaxy structure(e.g. Barnes 1990), change the characteristic size of the galaxy (Cox et al. 2006), strip stars into the ICM (Gallagher \& Ostriker 1972; Murante et al. 2004), and possibly add cold gas. Such processes may all contribute to the observed evolution of the red sequence.

In this work, we study passive galaxy growth from high redshift until today using cosmological hydrodynamic simulations. These simulations incorporate physicallymotivated but heuristic quenching mechanisms (described in Gabor et al. 2011) that yield a $z=0$ population of passive galaxies whose colour and luminosity distributions match observations. In particular, we include a prescription where we ensure that halos dominated by hot gas are continuously heated to keep circum-galactic gas hot. This simple and extreme prescription, approximating the effects of a heat source such as an AGN radio jet, successfully cuts off the fuel supply for star formation in massive halos which eventually yields passive galaxies. Although significant challenges remain for our favoured model, it provides a general qualitative guide for models where hot massive halos are the main drivers of passive galaxy formation. While Gabor et al. (2011) focused on the $z=0$ red galaxy population and compared various quenching mechanisms, here we focus on our most successful quenching mechanism and its implications for passive galaxy evolution from high redshift until today. Our model should not be considered physically correct in detail, but rather as broadly illustrative of the impact of some (unspecified) quenching mechanism that keeps hot halo gas hot on passive galaxy evolution over the past 10 billion years.

We begin by presenting an overview of our simulations in 2 . Then we present our results, beginning in $\$ 3$ where we examine number density and colour evolution, $\S 4$ where we look at how passive galaxies grow in mass and size via mergers, and $\S 5$ where we study mass and colour evolution versus environment. Finally, we summarize and discuss our conclusions in $\S 6$.

\section{SIMULATIONS}

\subsection{Simulation methodology}

We analyze the same cosmological hydrodynamic simulations described in Gabor et al. (2011). For completeness, we summarise the simulations here. They were run with an extended version of the N-body + smoothed particle hydrodynamics code GADGET-2 (Springel 2005). In addition to the basic N-body and hydrodynamics calculations, our version of the code includes sub-resolution modelling of gas cooling, star-formation, a model for chemical enrichment via AGB stars and both Type Ia and core-collapse supernovae, galactic winds associated with star-formation, and simple prescriptions for quenching star-formation.

We include both primordial and metal-line cooling assuming collisional ionisation equilibrium (Sutherland \& Dopita 1993), with the metallicities self-consistently tracked within simulations; see Oppenheimer \& Davé (2006, 2008) for details. We include heating from a metagalactic photo-ionising background (Haardt \& Madau 2001), assuming all particles are optically thin.

For star formation, we employ the two-phase model of Springel \& Hernquist (2003) based on the analytic description of McKee \& Ostriker (1977). Gas particles above a density threshold of $0.13 \mathrm{~cm}^{-3}$ are treated as cold, star-forming clouds embedded within a hot diffuse medium, and they form stars on a timescale consistent with the observed relation to surface gas density (Kennicutt 1998). Star-forming gas particles are converted into collisionless star particles stochastically, with a probability derived from the star formation rate.

As a gas particle is undergoing star formation, it selfenriches with metals from core-collapse supernovae. Furthermore, star particles share energy, mass, and metals with neighboring gas particles as a result of stellar mass loss from AGB stars and Type Ia supernovae. Stellar mass loss is calculated by assuming a Chabrier (2003) initial mass function, applying the stellar population models of Bruzual \& Charlot (2003, hereafter BC03), which account the mass lost by a stellar population at discrete times after the initial starformation event. We use Type Ia supernova rates from Scannapieco et al. (2006), and each such supernova results in the production of metals (mainly iron) that are shared with neighboring gas particles.

Our code further includes feedback in the form of galactic winds driven by star-formation (Oppenheimer \& Davé 2006, 2008). Just as a star-forming gas particle has some probability of being converted into a star particle, it has a probability of being kicked in a wind, which is given by $\eta$ (the mass loading factor) times the star formation probability. A wind particle is expelled from its host galaxy at a velocity $v_{w}$ typically a few hundred $\mathrm{km} \mathrm{s}^{-1}$. The velocities are chosen to match observations of local galaxy winds (Martin 2005; Rupke et al. 2005) and those seen at higher redshifts (e.g. Steidel et al. 2010). In particular, $v_{w} \propto$ the galaxy velocity dispersion, and $\eta \propto$ the galaxy circular velocity as calculated from an on-the-fly galaxy finder Oppenheimer \& Davé 2008); these scalings are predicted for momentum-driven winds (Murrav et al. 2005). A galaxy in a $10^{12} M_{\odot}$ halo at $z=1$ typically expels a wind with $v_{w} \approx 500 \mathrm{~km} \mathrm{~s}^{-1}$ and $\eta \approx 1.7$ (Oppenheimer et al. 2010). Once launched, 
winds are decoupled from the hydrodynamic calculation until they reach a density $10 \%$ of the critical density for star-formation, up to a maximum duration of $20 \mathrm{kpc} v_{w}^{-1}$. With this prescription, our simulations match a broad array of observational constraints on star-forming galaxies and the intergalactic medium (Oppenheimer \& Davé 2006, 2008; Oppenheimer et al.|2010; Davé et al. 2006, 2007; Finlator et al. 2007; Finlator \& Davé 2008).

Despite their broad success, winds driven by starformation generally do not result in the formation of red and dead galaxies. We incorporate an additional heuristic quenching model specifically to solve this problem (Gabor et al. 2011). For this model, we run a spherical overdensity algorithm on-the-fly to identify galaxy halos and distinguish gas above and below 250,000 Kelvin in each galaxy's halo. In halos with $>60 \%$ of all gas above this temperature cutoff, we apply constant heating (at every timestep) to all circum-galactic halo gas to force it to remain at the halo virial temperature. We exclude star-forming gas particles from this heating. Such heating could plausibly result from an AGN radio jet, but our model in fact uses more energy than thought to be available from observed AGN sources (Gabor et al. 2011). Other possible heat sources include cosmic rays (e.g. Mathews 2009) or graviational heating by infalling gas clumps (Dekel \& Birnboim 2008; Khochfar \& Ostriker 2008; Birnboim \& Dekel 2011). We remain agnostic about the exact mechanism, and instead examine whether an effective heating source can lead to a realistic population of quenched galaxies. We emphasize that our simulations do not track the growth of supermassive black holes.

The heating we apply to circum-galactic gas is sufficient to prevent it from condensing onto the galaxy, thus starving the galaxy of new fuel for star-formation. Over $1-2 \mathrm{Gyr}$, star-formation will exhaust any remaining cold gas, and starformation will cease. This model results in a bimodal colour distribution of galaxies, and red galaxy luminosity functions that match observations of the local universe. Although the model has some difficulties (like the required energy mentioned above), it should be a good representation of models where galaxies are quenched due to starvation enabled by their surrounding hot coronae.

\subsection{Runs and galaxy identification}

For this paper, we use a simulation of a $48 h^{-1}$ comoving Mpc random cosmological cube with $256^{3}$ dark and $256^{3}$ gas particles that incorporates all the above physics. We use a Wilkinson Microwave Anisotropy Probe concordance cosmology (Komatsu et al. 2009) with $H_{0} \equiv 100 \mathrm{~h}=70 \mathrm{~km}$ $\mathrm{s}^{-1} \mathrm{Mpc}^{-1}$, matter density $\Omega_{m}=0.28$, baryon density $\Omega_{b}=0.046$, a cosmological constant with $\Omega_{\Lambda}=0.72$, root mean square mass fluctuation at separations of $8 \mathrm{Mpc}$ $\sigma_{8}=0.82$, and a spectral index of $n=0.96$. Our simulation uses a gravitational softening length of $3.75 h^{-1} \mathrm{kpc}$. The initial gas particle mass is $1.2 \times 10^{8} M_{\odot}$, the typical star particle mass is half that, and our simulation results in $\sim 3000$ resolved galaxies at $z=0$. We have also used a simulation with $2 \times 384^{3}$ particles and the same volume to explicitly check that our main results are resolution-converged, as expected from previous work (Finlator et al. 2006; Davé et al. 2011). We note that high-resolution zoom simulations of individ- ual galaxies can produce quenched galaxies without explicit feedback, but they have too much stellar mass Naab et al. 2007).

We save snapshots of the simulation at 108 redshifts, starting at $z=30$ and ending at $z=0$. The time between snapshots ranges from a few tens of Myrs at high redshift to $\sim 300$ Myr at low redshift. The snapshots contain information for simulation particles, such as position, velocity, mass, metallicity, gas density, gas temperature, and star-formation rate. From these particle data, we determine galaxy properties to compare with observables.

We use Skid 1 to identify galaxies (cf. Gelb \& Bertschinger 1994; Kereš et al. 2005). Skid provides a list of member particles (star and star-forming gas) for each simulated galaxy. The sum of member star particle masses is then the galaxy stellar mass, and the instantaneous star formation rates of the gas particles are summed to give the star formation rate of the galaxy.

We then calculate galaxy spectra using the models of Bruzual \& Charlot (2003), as in Finlator et al. (2006). We treat each star particle as a single stellar population with an age and metallicity determined directly in the simulation. By adding up the spectra of all star particles within a galaxy, we obtain the spectrum of that galaxy, from which we measure galaxy colours and magnitudes in various bands. Since we focus on the passive galaxy population that is thought to be mostly dust-free (Lauer et al. 2005), we ignore dust reddening and extinction.

\section{$2.3 \quad$ Building merger trees}

Beyond knowing galaxy properties at a given redshift, we wish to study the histories of individual galaxies as they evolve. For this we must connect each galaxy at $z=0$ to its progenitor galaxies at earlier redshifts: i.e., build a merger tree. We do so by determining, for every star particle in a given galaxy, which galaxy from an earlier snapshot that star particle lived in. If the star formed recently it wouldn't have lived in any previous galaxy. By tracing star particles in this way, we determine the immediately preceding progenitors of each galaxy. Galaxies with multiple progenitors must have undergone a merger (or at least some galaxy interaction, such as stellar stripping).

Creation of a merger tree from a cosmological simulation involves handling a number of pathological circumstances owing to the stochastic nature of the gas and star particles. A common annoyance is that if two galaxies fly by each other in a close encounter, Skid (and most other galaxy finders) will often identify them as a single galaxy during one or a few timesteps, but then again as separate galaxies at later times. Sometimes star particles are not assigned to any group. While these issues do not affect the majority of galaxies and hence do not strongly impact the overall results, they must nevertheless be handled in an appropriate manner.

To surmount these difficulties, we follow strategies described in Maller et al. (2006), who dealt with the same issues. If a star particle is not assigned to any Skid groups at a given timestep, we trace its history through earlier timesteps

\footnotetext{
1 http://www-hpcc.astro.washington.edu/tools/skid.html
} 
Gabor et al.

to find the last galaxy it was in, and assign it to the descendant of that galaxy. When two Skid groups at one timestep have a single common progenitor at an earlier timestep, we assume that the two groups are a merging pair and assign them to the same galaxy.

The above prescription assumes that once galaxies are joined by Skid, they will eventually merge. But some fly-by interactions will not lead to mergers. To account for this, we identify galaxies at $z=0$ that are composed of multiple Skid groups and separate those groups into distinct galaxies. We find the earliest timestep when both groups' stars are assigned to the same galaxy, and from then on assign a new galaxy ID to those stars in the smaller of the two groups. Once every star particle is assigned to a galaxy at every timestep, we have the full merger history.

\subsection{Environment measures}

In order to study where red galaxies emerge in our simulation, we measure the local galaxy density around Skid galaxies. We use two density measures. An intuitive and simple approach is to count the number of simulated galaxies within $1 h^{-1} \mathrm{Mpc}$ of the galaxy of interest (Blanton 2006). For some purposes this measure is too noisy, as it places galaxies into discrete bins of density, and some galaxies have few neighbors within $1 h^{-1} \mathrm{Mpc}$. Thus, as an alternative we use Smooth 2, which calculates the mean density at each galaxy's location after smoothing over the nearest 16 galaxies with a spline kernel (similar to an SPH density calculation). This allows us to quantify low densities less stochastically than by simply counting galaxies within some fixed distance. In some cases we use a galaxy's local overdensity measured relative to the mean resolved galaxy density in our simulation volume. Based on numerous tests, our density estimator based on Smooth is comparable to and correlated with common observable density estimators such as a 5th-nearest neighbor density (e.g. Kovač et al. 2010).

\section{EVOLUTION IN COLOUR AND NUMBER DENSITY}

\subsection{Color-mass diagrams and stellar mass functions}

In this section we examine how colours and number densities (parameterized via the galaxy stellar mass function) evolve with redshift in our simulations. We note that our simulations do not perfectly match present-day observations of passive galaxies, as detailed in Gabor et al. (2011). For instance, the colors are slightly too blue and the red sequence does not have the correct slope, owing to an underproduction of massive galaxies' metallicities by $\sim 50 \%$ (see Gabor et al. 2010, 2011, for a full discussion). Nonetheless, our simulations clearly identify two general trends in passive galaxy evolution. First, passive galaxies emerging at $z>2$ are at the massive end of the galaxy stellar mass function, followed shortly by small satellites, then finally the intermediate region of $10^{10} M_{\odot}$ galaxies. Second, the most massive red galaxies in our model grow substantially at late times.

\footnotetext{
${ }^{2}$ http://www-hpcc.astro.washington.edu/tools/smooth.html
}

Figure 1 shows colour-mass diagrams and galaxy stellar mass functions for simulated galaxies at redshifts $0,0.3,1$, and 2. The $g-r$ colours are rest-frame SDSS filter bands, and do not include any correction for obscuration due to dust these are intrinsic colours. Dust reddening can induce large changes in galaxy colors (see Gabor et al. 2010), but for a given simulated galaxy the dust reddening is highly uncertain. We thus focus on the more robust intrinsic properties of simulated galaxies.

Our quenching model leads to an obvious bimodality in (intrinsic) galaxy colors, which corresponds to a bimodality between star-forming and quiescent galaxies. We divide the galaxy population into blue and red using a straight line in $g-r$ colour versus $r$ magnitude space in the same way as in Gabor et al. (2011). We evolve the normalization (but not the slope) of our colour separation in a simple linear way that approximately accounts for passive evolution: $y_{\mathrm{sep}}(z)=y_{\mathrm{sep}, \mathrm{z}=0}-0.1 z$. Here $y_{\text {sep }}$ is the $\mathrm{y}$-intercept (i.e. colour) of the line of separation, and $z$ is the redshift. The hatched region on the left of the figure denotes the mass range of galaxies that may be inadequately resolved. The colour-mass diagrams show strong growth in the passive galaxy population from $z=2 \rightarrow 0$. Both the blue and red galaxy populations become redder with time, at roughly the same rate.

The lower set of panels of Figure 1 shows galaxy stellar mass functions separated into blue (dashed line) and red (solid line) populations. We plot mass function observations taken from Drory et al. (2009) and Brammer et al. (2011), who likewise split galaxies into star-forming and quiescent populations (blue vs. red points). Since dust reddening adds difficulty and complexity to the separation of star-forming and quiescent galaxies (and since we use different separation criteria), we emphasize that comparisons with observations are only qualitative. We note that Brammer et al. (2011) paid particular attention to separating truly passive from dusty star-forming galaxies.

The simulated blue galaxy stellar mass function evolves only weakly with redshift, but the red galaxy mass function undergoes drastic growth from $z>2$ to 0 . The buildup of passive galaxies reflects the quenching of star-forming galaxies due to starvation in our model (see Gabor et al. (2011)). The red sequence grows from mostly non-existent at $z \sim 3$ to dominating the galaxy population at $z \sim 0$. The mass functions clearly show that the most massive galaxies are the first to become red, followed by low-mass galaxies that turn out to be satellites to the massive ones. This directly results from the fact that the most massive galaxies in our simulations are the first to form a corona of hot gas. Massive galaxies have massive dark matter halos, and only massive dark matter halos form stable hot gas coronae Birnboim \& Dekel 2003).

\subsection{A dearth of passive galaxies at $M_{*} \sim 10^{10} M_{\odot}$}

Examination of Figure 11 reveals a characteristic "dip" in the simulated red galaxy mass function at $M_{*} \approx 10^{10} M_{\odot}$, which persists at all redshifts but is more prominent at high redshifts. The red mass function peaks at high masses $M_{*} \approx$ $10^{10.6} M_{\odot}$, declines to intermediate masses $M_{*} \approx 10^{10.0} M_{\odot}$, and then rises again to low masses $M_{*} \lesssim 10^{9.8} M_{\odot}$.

The first passive galaxies form at the massive end by 


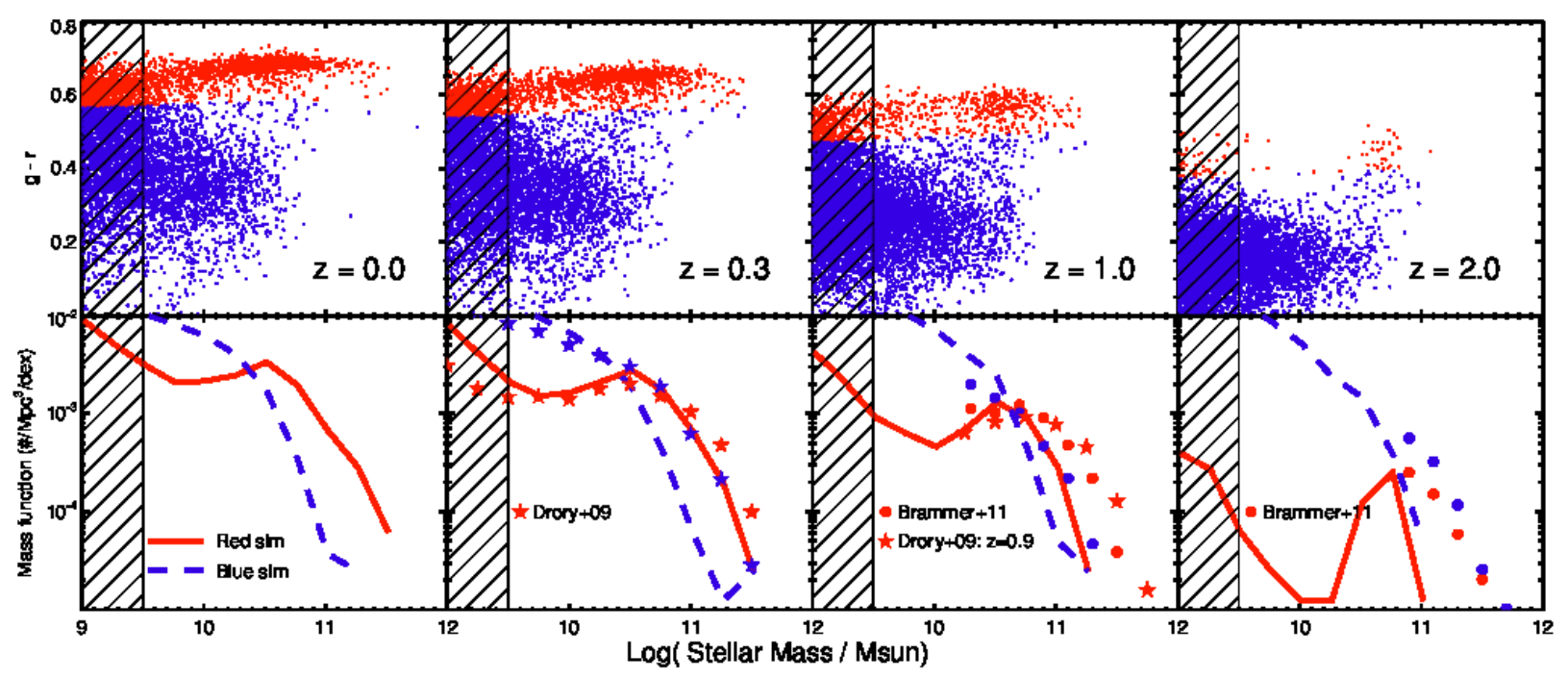

Figure 1. Redshift evolution of colour-mass diagrams (top row) and galaxy stellar mass functions (bottom row). Redshift increases from left to right, as labeled. Red and blue galaxies are separated using a redshift-dependent cut, as illustrated by the red and blue colour-coding in the CMD. In the mass function panels, solid lines denote the simulated red sequence, dashed lines the simulated blue cloud, and observed data points are taken from the literature. The hatched region indicates poorly resolved galaxies. Massive red galaxies are present at $z=2$, but not in sufficient numbers compared to observations. There is a dearth of intermediate mass galaxies $\left(\sim 10^{10} M_{\odot}\right)$ that is more pronounced at higher redshifts.

$z \approx 3$ (which is an underestimate since our limited simulation volume does not probe the most massive structures at $z>3$ ). Soon thereafter, small satellites of the first quenched galaxies become red as well, owing to starvation of gas in growing hot halos (Simha et al. 2009). Intermediate-mass galaxies with $M_{\text {stellar }} \approx 10^{10} M_{\odot}$ are the last to quench, filling in the gap between the centrals and satellites such that by $z \sim 0$ this bimodality within the red sequence is not so evident. Therefore, a strong prediction of this quenching mechanism is that there is a distinct gap between the massive and low-mass ends of the red sequence that becomes more pronounced with redshift.

A variety of observational studies already suggest that there are fewer red galaxies at intermediate-mass than at the high-mass peak, especially at high redshift, as our simulated mass functions imply (Stutz et al. 2008; Drory et al. 2009; Mortlock et al. 2011; Rudnick et al. 2009; Yang et al. 2009; Rudnick et al. 2012; Tal et al. 2012). At $z \approx 0$, recent studies show a slight decline in the red galaxy mass function from high to intermediate masses, requiring a characteristic double-Schecter function to get a good fit (Yang et al. 2009; Peng et al. 2010; Baldry et al. 2008, 2012). Unlike our simulation, however, observations do not typically show an upturn toward lower masses (but see Drorv et al. 2009; Yang et al. 2009). Some semi-analytic models show an analogous mass bimodality for elliptical galaxies (De Lucia et al. 2012), although the driving processes are likely different.

Although further observations will be required to confirm that there are more high-mass than intermediate-mass quenched galaxies (especially at higher-redshift), this trend naturally emerges from our quenching model. Our simple model, where hot gas dictates the formation of the red sequence, provides a compelling physical picture, which we describe next.

\subsection{Central and satellite evolution along the red sequence}

The separation in mass range between central and satellite galaxies along the red sequence is more pronounced at earlier epochs, leading to a sort of bimodality along the red sequence itself. This is naturally understood within a hierarchical structure formation model, as we outline here.

In our model, the initial emergence of the massive red sequence is directly tied to the requirement that a galaxy's halo be dominated by hot gas for quenching to begin. Galaxy evolution models have long predicted that gas in the haloes of galaxies should be virialised and thus hot (Rees \& Ostriker 1977; Silk 1977; White \& Rees 1978). Gas in haloes with masses below a few $\times 10^{11} M_{\odot}$, however, will have short cooling times, so that it never reaches the virial temperature of the halo (Binnev 1977; White \& Frenk 1991; Birnboim \& Dekel 2003; Kereš et al. 2005). In our simulations with metal-line cooling the threshold for a hot gasdominated halo is $\sim 10^{12} M_{\odot}$ (Gabor et al. 2010), with little or no redshift evolution.

The key assumption in our model is that in such hotgas dominated halos, gas is very inefficiently deposited onto the galaxy, likely owing to the contribution of some preventive feedback process(es) like AGN heating (e.g. Dekel \& Birnboim 2006; Croton et al. 2006; Cattaneo et al. 2006; Bower et al. 2006; Birnboim et al. 2007). Since galaxy stellar masses are well-correlated with halo masses below the cutoff for quenching, only central galaxies with the highest stellar masses will live in hot halos at early times.

As the universe evolves and individual halos grow, more galaxy halos become massive enough to support hot coronae. Massive central galaxies thus continue to move onto the red sequence. Our hot gas fraction cutoff of $60 \%$ roughly corresponds to a stellar mass of about $10^{10.5} M_{\odot}$, though there 
is significant scatter. Once galaxies exceed this stellar mass, they will tend to migrate toward the red sequence over the span of $1-2$ Gyr. We note that this mass cutoff is unlikely to be universal at $z \gtrsim 2$, since there are seen to be a number of star-forming galaxies with stellar masses approaching and even exceeding $10^{11} M_{\odot}$ (e.g. Daddi et al. 2007). These galaxies may be fed by cold streams that are dense enough to penetrate the hot halo (Dekel et al. 2009).

What happens with satellite galaxies? When a central galaxy becomes quenched by its own hot corona, its satellites are likely to be embedded within that hot corona as well. Based on our criteria for quenching, these satellites will be quenched as well. In LCDM, the most massive subhalo is typically less than $10 \%$ of the mass of its parent halo (Gao et al. 2004) - when a central galaxy of mass $10^{10.5} M_{\odot}$ is quenched, its satellites will usually be $<10^{9.5} M_{\odot}$. This creates a "gap" where there are no red galaxies around $10^{10} M_{\odot}$.

As time progresses, massive quenched halos merge with nearby halos owing to hierarchical growth. For example, a star-forming galaxy with a mass of $M_{*}=10^{10} M_{\odot}$ may fall into a more massive, group-sized halo. As the smaller halo falls into the region dominated by hot gas, its central galaxy becomes a satellite and is quenched by the hot ICM on a $1-2$ Gyr timescale (Simha et al. 2009; Skibba 2009; Wetzel et al. 2011). These infalling (former) central galaxies begin to populate the intermediate-mass portion of the red sequence. Therefore hierarchical growth combined with starvation by the hot corona results in a gradual "filling in" of the intermediate-mass red sequence. These trends will be explored in more detail, particularly in relation to environment, in 5

In summary, our model predicts that galaxies with $M_{\text {stellar }} \sim 5 \times 10^{10} M_{\odot}$ are the first to become passive. Galaxies in the "gap" at $M_{\text {stellar }} \sim 10^{10} M_{\odot}$ catch up with time. The key feature driving these trends is that our quenching mechanism, tied to the hot gas coronae that form in halos $\sim 10^{12} M_{\odot}$, selects a characteristic mass for passive galaxies. That is, once a star-forming galaxy achieves a mass $\gtrsim 10^{10.5} M_{\odot}$, it will become quenched and move to the red sequence. Less massive galaxies are only quenched as satellites (or nearby halos) of more massive halos. More massive red galaxies can only be obtained via merging. While quantitative details may vary with parameter choices, these are generic outcomes for hierarchical quenching models keyed to a critical halo mass.

\subsection{Paths to the red sequence}

Here we focus on the path of individual galaxies in colourmass space: Given a red galaxy at $z \sim 0$, what path did it take to get there? It turns out that massive galaxies in our models follow paths much like those in the schematic diagram of Faber et al. (2007): they move onto the red sequence at masses near the turnover in the mass function, then grow further through mergers and accretion of satellite galaxies.

In Figure 2 we show the paths of three typical galaxies (coloured lines) in the colour-mass diagram. We plot a background of simulated galaxies at $z=0$ (points), and we correct for passive evolution of colours for the galaxy paths

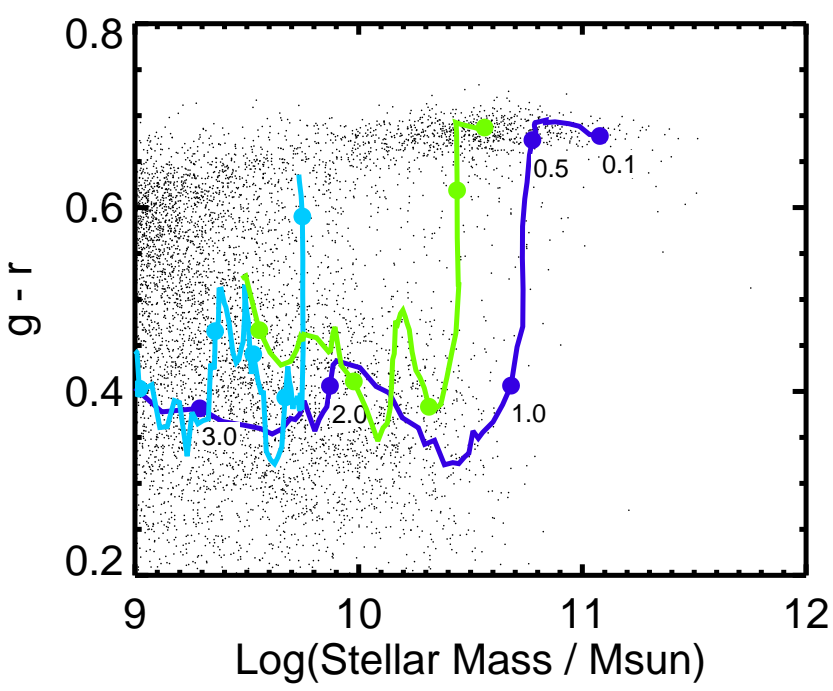

Figure 2. Example evolutionary paths in the colour-mass diagram. We show the paths of three galaxies (coloured lines) as they evolve through the colour-mass diagram, as represented by $z=0$ galaxies (small points) in the background. This is analogous to the schematic diagram of Faber et al. (2007). Dots indicate redshifts along the evolutionary paths, as labelled for the most massive galaxy. Galaxies grow in stellar mass along the blue sequence, then move vertically to the red after quenching. Once on the red sequence, galaxies grow only via mergers and accretion of satellites. Such growth can be substantial, as for the path of the most massive galaxy shown.

in the same way that we evolved our line separating blue and red galaxies 3.1 . We do not include dust reddening.

All three of these galaxies build stellar mass via starformation for several Gyrs before moving onto the red sequence. Once quenched, they stop building stellar mass and cross vertically and rapidly over the green valley to the red sequence in $\lesssim 2$ Gyr (see the star-formation histories in Gabor et al. 2011). The least massive galaxy makes this transition at late times $(z \sim 0.1)$, and does not change in mass or colour after reaching the red sequence because it does not have time to undergo mergers. The most massive galaxy, in contrast, reaches the red sequence at $z \sim 0.5$ when its mass is several $\times 10^{10} M_{\odot}$, and then continues to grow in mass along the red sequence via mergers, eventually obtaining a mass of $\sim 10^{11} M_{\odot}$.

More broadly, these paths are fairly representative for galaxies in their respective mass ranges. Low-mass galaxies tend to move onto the red sequence at late times, and grow little after doing so, while massive galaxies become passive at earlier epochs and grow in mass by merging with smaller galaxies.

\subsection{Specific star formation rates}

A key observational constraint on the transition of galaxies onto the red sequence is provided by the distribution of specific star formation rates (sSFR) versus stellar mass. This contains similar information to the colour-mass plots above, but here we additionally compare our current quenching sim- 


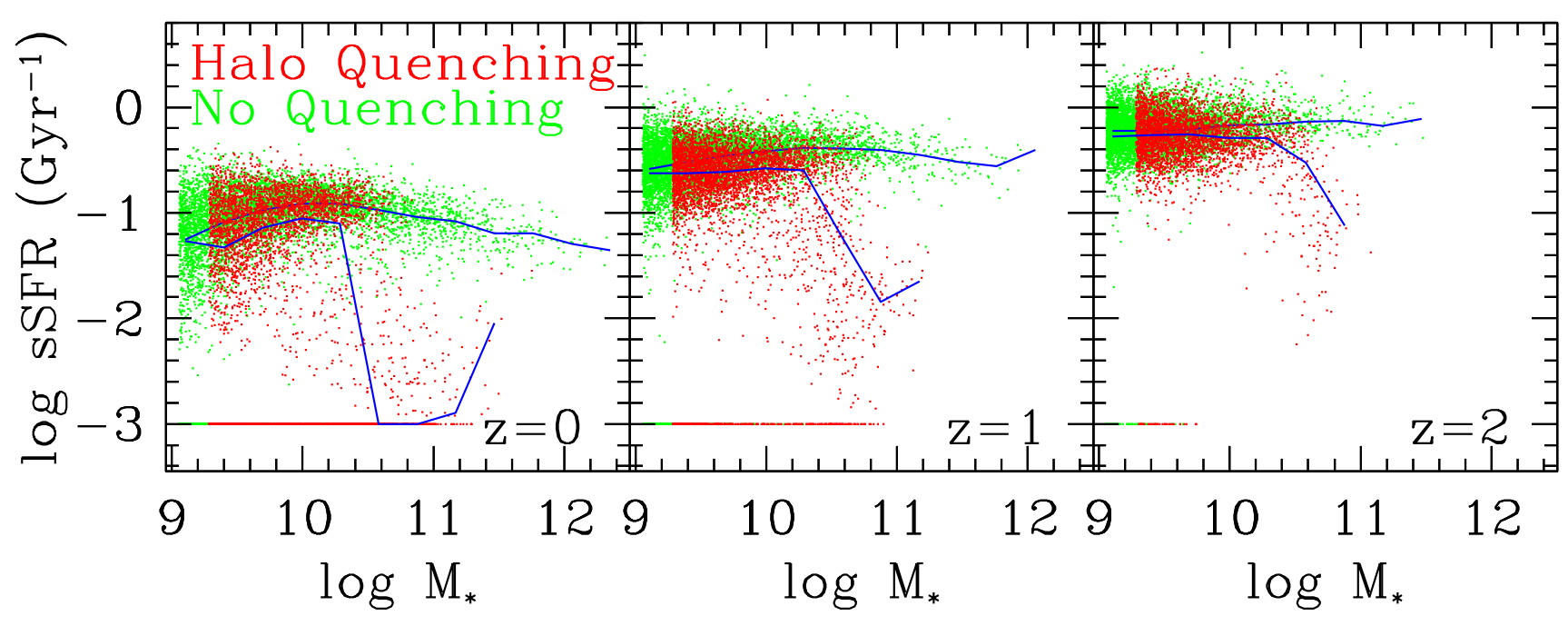

Figure 3. Specific star formation rate (sSFR) as a function of stellar mass at redshifts 0 (left), 1 (middle), and 2 (right). Red points correspond to our halo quenching model and green points to a simulation without quenching. Blue solid lines show the running median sSFR for both models. Many quenched galaxies have no star-formation, so we assign a minimum sSFR of $10^{-3} \mathrm{Gyr}^{-1}$. Quenching has little impact on the sSFR- $M_{*}$ relation below $\sim 10^{10.5} M_{\odot}$, but causes a drop in sSFR above this threshold at all redshifts. The transition quenching mass is blurred by satellite galaxies $\sim 10^{10} M_{\odot}$ becoming quenched.

ulations with previous similar runs without quenching from Davé et al. (2011). We particularly examine the stellar mass range over which the transition to passive occurs, since the observed lack of a sharp delineation in stellar mass between passive and star-forming galaxies has been used as an argument against a quenching prescription based on halo mass (as ours is, approximately).

Figure 3 shows sSFR as a function of stellar mass for a simulation without any quenching (green points) and the halo quenching run presented in this paper (red). Blue lines show a running median for each. Galaxies with sSFR $\leqslant$ $10^{-3} \mathrm{Gyr}^{-1}$ are shown along bottom of the plot. The noquenching run employs the same cosmology, volume, input physics (besides quenching), and outflow model, but it has somewhat better resolution $(\sim \times 3)$.

The form and evolution of the sSFR for the nonquenched case is discussed extensively in Davé et al. (2011). Key points are that the relation is flat at high- $z$ moving towards a mildly negative slope by $z \sim 0$, sSFR increases with redshift at a given stellar mass steadily out to $z=2$, and the scatter is typically $\sim 0.2$ dex or less. The relation fundamentally arises from gravitationally-driven smooth cold accretion that dominates galaxy fueling, and diminishes with time as the Universe expands (e.g. Davé 2008; Cen 2011).

The impact of halo quenching is clearly seen at masses $\gtrsim 3 \times 10^{10} M_{\odot}$, the same mass where many galaxy properties are observed to undergo a strong transition (Kauffmann et al. 2004). Below this transition mass, there is a minimal difference $(<0.2 \mathrm{dex})$ in the quenched and non-quenched relations. Above this transition mass, the sSFR drops quickly towards small values, which is qualitatively similar to observations (e.g. Salim et al. 2007). The transition mass evolves very little with redshift, being only marginally higher at $z \sim 2$. The highest mass galaxies are still forming a "frosting" of stars at a much reduced yet non-trivial rate (Trager et al. 1998, 2008), reflecting the in- fall of satellites that are not fully quenched. In our model there are very few galaxies above the transition mass on the main star-forming sequence, in conflict with observations. This conflict is also found in SAMs with similar quenching mechanisms (Somerville et al. 2008).

Particularly noteworthy are the intermediate-mass galaxies that we argued earlier are "filling in" the gap of the red sequence at stellar masses of $\sim 10^{10} M_{\odot}$. As these galaxies become more numerous towards lower redshifts, the transition between quenched and unquenched is significantly blurred, such that many quenched galaxies are present at masses below the nominal transition mass. Hence the argument that the observed gradual transition from unquenched to quenched galaxies rules out a model based on halo mass quenching (which our model approximates) may not be justified. While our simulations qualitatively resemble observations, it is worth noting that such environmental quenching processes are not as robustly modeled as we would like in our simulations, and hence a more quantitative comparison will have to await improved modeling of the interactions of galaxies moving through hot halo gas.

\section{GROWTH ALONG THE RED SEQUENCE}

\subsection{Mergers since quenching}

We have seen that a galaxy does not stop growing and evolving once it reaches the red sequence (see Figure 2). Its existing stellar population ages, that population sheds mass via stellar winds, and it obtains new stars (and gas) via mergers. Here we show that the most massive galaxies, which are quenched at early times $(z>1)$, often grow by factors of a few between quenching and the present day. In contrast, low-mass red galaxies (mostly satellites) tend to lose mass 

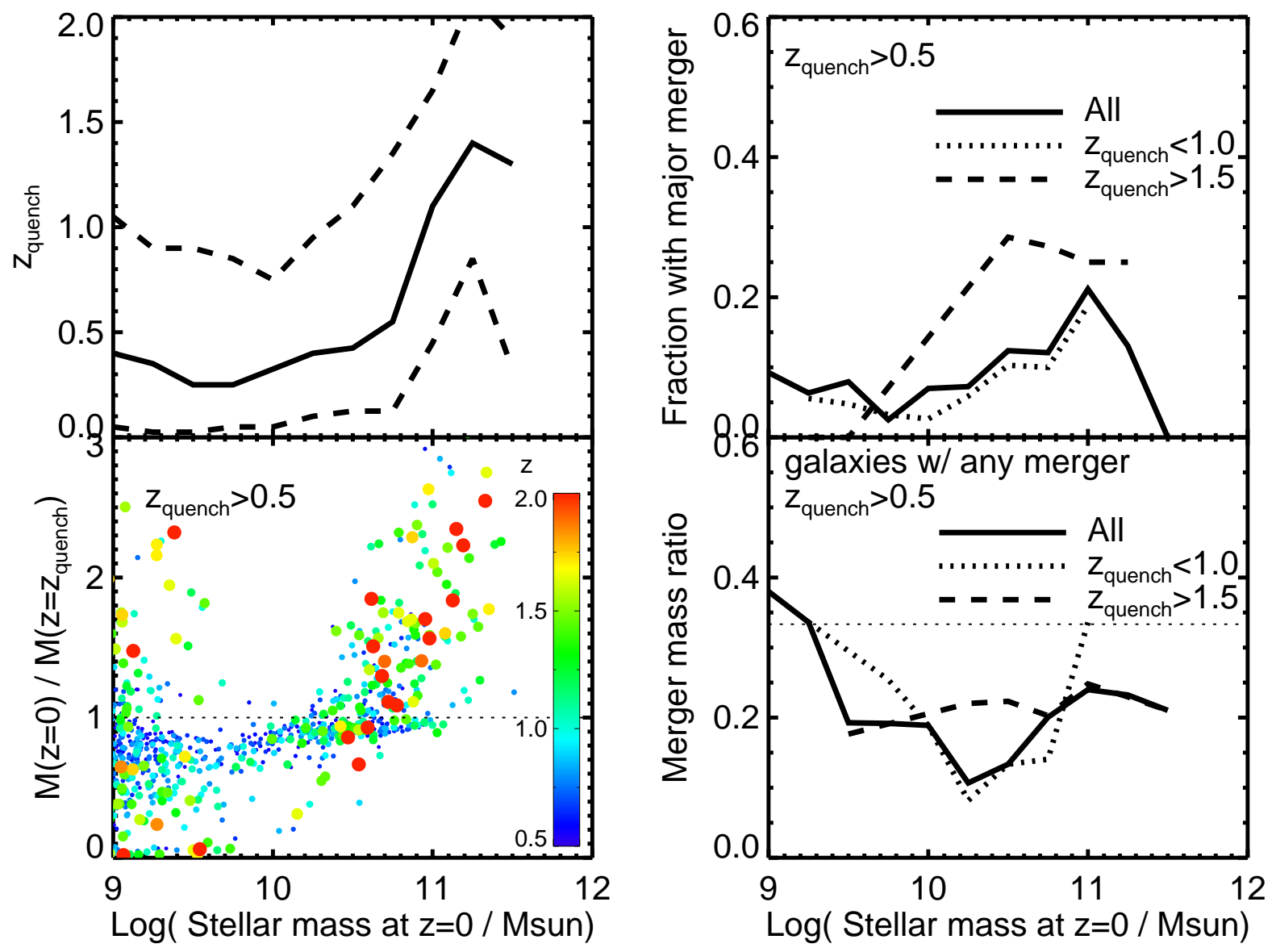

Figure 4. Growth of galaxies along the red sequence. In our model, the most massive galaxies typically grew by factors of a few since being quenched at $z \sim 1$, and that mass growth is dominated by minor mergers. Top Left: median (solid line), 10th and 90th percentiles (dashed) of the redshift at which red sequence galaxies were quenched $\left(z_{\text {quench }}\right)$ in different mass bins. Bottom Left: fractional mass growth of red sequence galaxies since $z_{\text {quench }}$, as a function of stellar mass at $z=0$, among galaxies quenched before $z=0.5$. Galaxy points are colour-coded by $z_{\text {quench }}$, and galaxies with higher $z_{\text {quench }}$ have larger symbol sizes. Top Right: the fraction of galaxies per bin that have undergone at least one major merger since being quenched, among galaxies with $z_{\text {quench }}>0.5$. The fraction for galaxies with $0.5<z_{\text {quench }}<1.0$ (dotted) is typically lower than that for galaxies quenched earlier, $z_{\text {quench }}>1.5$ (dashed). Bottom Right: the mass-weighted average merger mass ratio, among galaxies with $z_{\text {quench }}>0.5$. The mean merger is always a minor merger $(<1 / 3$, thin dotted line). Galaxies quenched at high redshifts (dashed line) all have a merger mass ratio of $\approx 0.2$, while those quenched at somewhat lower redshifts (dotted) show stronger variation with mass.

through stellar evolution and stripping without undergoing significant mass growth via mergers.

The top left panel of Figure 4 shows the distribution of the redshift at which galaxies are quenched, $z_{\text {quench }}$, as a function of the final stellar mass at $z=0$. We plot the 50 th (solid), 10th, and 90th percentiles (dashed) of the distribution in each bin. The quenching redshift is defined as the redshift when a galaxy first appears red in the colourmagnitude diagram, with an evolving dividing line between red and blue galaxies as described in 3.1 Low-mass galaxies on the $z=0$ red sequence, which are predominantly satellites, are quenched at fairly late times (generally $z<1$ ). Massive galaxies $\gtrsim 10^{11} M_{\odot}$ are quenched earlier, typically at $z \gtrsim 1$ and beyond.

The bottom left panel of Figure 4 shows, as a function of the $z=0$ stellar mass, the fractional mass growth of red sequence galaxies between the redshift of quenching and the present day, $y \equiv M_{\text {stellar }}(z=0) / M_{\text {stellar }}\left(z=z_{\text {quenched }}\right)$. Since many galaxies are quenched at late times, and such galaxies have not had time to undergo mergers, we only plot the mass growth for galaxies with $z_{\text {quench }}>0.5$. This gives a long enough time baseline $(\sim 5 \mathrm{Gyr}$, much longer than the halo dynamical times) to assess red galaxy growth via mergers. Galaxy points are colour-coded by $z_{\text {quench }}$, as indicated on the right side of the panel.

Departures from the $y=1$ line (i.e. no change in stellar mass, dotted line) increase with quenching redshift: The blue points that are the most recently quenched galaxies typically lie near $y=1$, and in fact are slightly below owing to stellar mass loss. At higher quenching redshifts, galaxies span a wider range in $y$. In some rare cases, galaxies lose mass catastrophically by tidal interactions with other galaxies, and end up near $y=0$. A significant proportion of galaxies quenched at earlier times with $M>10^{10.5} M_{\odot}$, how- 
ever, have grown substantially, by factors up to $\sim 3$. Since these massive galaxies live in high-density environments, and they've been quenched for longer, they have had more opportunities to acquire mass via mergers than their lower-mass counterparts. Hence $y$ reflects the amount of mass growth by mergers in massive galaxies since the time of quenching, and in fact underestimates the growth since stellar mass loss also occurs. We note that a small number of galaxies become quenched at $z<0.7$ yet undergo mass growth by $>\times 2$ via several major mergers. Some low-mass galaxies with $y>2$ are only marginally resolved in our simulation.

The top right panel of Figure 4 shows the fraction of $z=0$ red sequence galaxies that have undergone at least one major merger (1:3 mass ratio or larger) since being quenched. Post-quenching major mergers are generally rare, happening in typically only $\sim 10 \%$ of galaxies, and up to $\sim 20 \%$ of galaxies with $M_{*} \sim 10^{11} M_{\odot}$. Galaxies quenched at the earliest times have major merger fractions as high as $30 \%$. Semi-analytic models indicate major merger fractions reaching $>50 \%$ for massive galaxies (De Lucia et al. 2006; Wang \& Kauffmann 2008). These studies, unlike ours, have considered the integrated merger history of the model galaxies. Since mergers are more common at higher redshifts (e.g. Hopkins et al. 2010b), restricting ourselves to mergers since the time of quenching naturally leads to a lower fraction of galaxies with at least one merger. In our model, major mergers play a sub-dominant role in the overall growth of massive red galaxies.

With little or no in situ star formation, the growth of red galaxies must therefore owe predominantly to minor mergers. The bottom right panel of Figure 4 shows the mean merger mass ratio as a function of $z=0$ stellar mass. By weighting each merger by its mass, we can assess the contribution that mergers of a given mass ratio make to the overall growth of passive galaxies. The typical merger mass ratio for red sequence galaxies in our simulations is $\sim 0.2$ or $1: 5$, below the commonly-used 1: 3 cutoff for major mergers (thin dotted line). This typical value agrees with that obtained in higher-resolution individual galaxy re-simulations by Oser et al. (2012). At all stellar masses, red galaxy mass growth is dominated by minor mergers.

In summary, massive red sequence galaxies in our simulation grow by factors of up to several in mass between the time they are quenched (typically $z \sim 1$ ) and redshift 0 . Lower mass passive galaxies (both satellites and centrals) tend to be quenched at later epochs, and grow less, typically even diminishing in mass owing to stellar evolution. Mass growth at all masses is dominated by minor mergers with a characteristic ratio of $1: 5$, with at most a $30 \%$ contribution from major mergers.

\subsection{Size evolution}

Observations indicate that massive passive galaxies are more compact at high redshifts than at $z \sim 0$ by factors of at least 2 and possibly up to 6 (Toft et al. 2007; van der Wel et al. 2008; van Dokkum et al. 2008; Trujillo et al. 2007, 2011). While observational biases presented an initial concern, recent work suggests that much of the evolution must be physical, and probably arises from a combination of several physical processes. These processes include adiabatic expansion associated with stellar mass loss, major

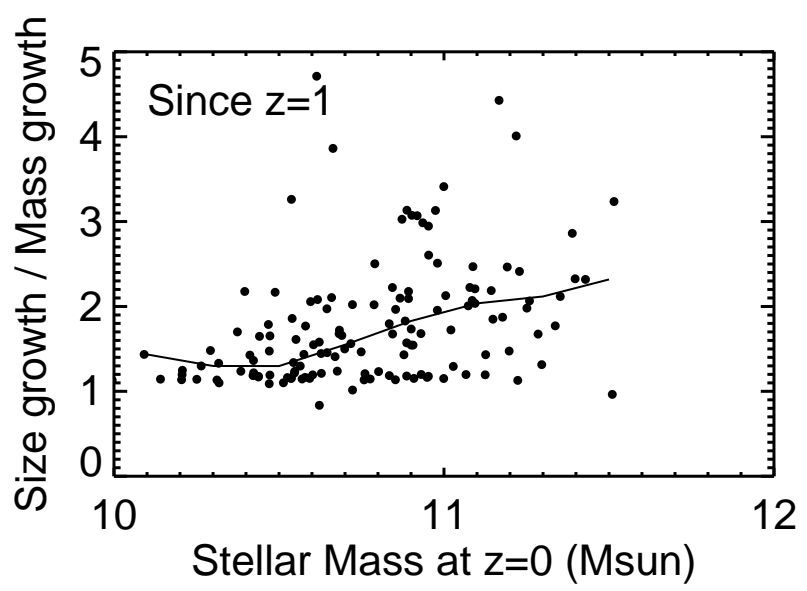

Figure 5. The ratio of size growth to mass growth for red sequence galaxies, since $z=1$, as a function of their stellar mass at $z=0$. A solid line indicates the median. More massive galaxies show more size growth than lower-mass ones. In some rare cases the galaxy size may evolve several times $(\sim 3)$ more than the mass. This is roughly in line with observations, where the ratio is typically $\sim 2$ for evolution since $z \sim 2$.

mergers, minor mergers, and evolution in mass-to-light ratios (e.g. Bovlan-Kolchin et al. 2006; Naab et al. 2009; Bezanson et al. 2009; Hopkins et al. 2010a).

Our simulations make specific predictions about the distribution of merger mass ratios that contribute to red galaxy growth. Therefore, even though the spatial resolution of our simulations is not well-suited to study the internal structure of individual galaxies, we can use simple analytic mergerbased estimates to approximate the size growth that our simulated galaxies might undergo.

We construct a simple model for the effects of mergers following the formalism presented in Naab et al. (2009), which arises directly from the virial theorem plus energy conservation. The model is appropriate for a purely collisionless system, so we implicitly ignore any effects of dissipation - since this model concerns quenched galaxies this is a reasonable simplification, but it may lead to an overestimate of the size growth due to mergers. Given a stellar accretion event (i.e. a merger) of mass $M_{a}$ onto a galaxy of mass $M_{g}$, the ratio of final to initial size of the galaxy is given by:

$\frac{r_{f}}{r_{i}}=\frac{(1+\eta)^{2}}{1+\eta \epsilon}$.

Here, $\eta=M_{a} / M_{g}$ is the fractional mass increase, and $\left.\epsilon=\left\langle v_{a}^{2}\right\rangle /<v_{g}^{2}\right\rangle$ is the ratio of the two galaxies' stellar populations' mean square velocity dispersions. Using highresolution simulations, Naab et al. (2009) has shown that this formula accurately describes the size evolution of earlytype galaxies undergoing hierarchical mergers.

To estimate size growth, we must therefore estimate the mass ratio and the velocity dispersion ratio between the satellite (i.e. less massive) and central (more massive) galaxies. We know the ratio of stellar masses directly from our merger tree. For the velocity dispersion, since we do not accurately resolve it in our simulations (Oppenheimer \& Davé 
2008), we instead employ an observational result relating it to stellar mass from Gallazzi et al. (2006): $\log \sigma=$ $-0.895+0.286 \log M_{\text {stellar }}$. With this relation, we obtain $\epsilon=\left(\sigma_{a} / \sigma_{g}\right)^{2}=\left(M_{a} / M_{g}\right)^{0.57}$, which gives comparable results to the theoretical relation with an exponent of $2 / 3$ at any redshift (Mo et al. 1998). Thus, for each merger event, we can calculate the fractional size growth of the remnant galaxy. Taking each $z=0$ red galaxy, we consider its entire merger history since being quenched. We account for the size growth of each of its merger events and multiply them to find the galaxy's total fractional size growth (although we cannot determine whether the galaxies start out small as observed).

We show the results of this exercise in Figure 5 which shows the size growth relative to mass growth $\left(r_{f} M_{i} / r_{i} M_{f}\right)$ as a function of stellar mass. We plot this quantitiy instead of merely size growth because massive red galaxies in our simulations may not grow the correct amount of stellar mass, as hinted by the mass functions in 3.1. The size growth relative to the mass growth of galaxies is a reflection of the prevalence of minor vs. major mergers.

Based on this simple modeling, minor mergers appear to be numerous enough to drive factors of several in size growth of passive galaxies over time. Galaxies with stellar masses $>10^{11} M_{\odot}$ show typical size growth a factor of 2 greater than their mass growth, indicating that a series of minor mergers tends to puff up these galaxies, as argued by Naab et al. (2009). Lower mass galaxies have not grown as much since moving onto the red sequence, since as we showed in Figure 4 they undergo less merging. These results, using a simple analytic model, are broadly consistent with the idea that minor mergers drive the rapid size growth seen in massive red galaxies since high redshift.

In summary, a simple model of red galaxy size growth driven by mergers shows that massive galaxies with $\gtrsim$ $10^{11} M_{\odot}$ in our simulation typically have grown in size by a factor of 2 relative to their mass growth since $z=1$. The degree of size growth increases with stellar mass. These results are broadly consistent with observations as well as previous simulation results.

\section{ENVIRONMENTAL FACTORS}

\subsection{Bivariate dependence of environment on colour and $M_{\text {stellar }}$}

We have shown that the massive and low-mass ends of the red sequence grow first in our model - not until $z \lesssim 0.3$ do intermediate-mass galaxies around $10^{10} M_{\odot}$ appear in comparable numbers along the red sequence. In this section, we examine how environmental factors drive this behaviour.

We illustrate these environmental effects in Figure 6 On the left we show a colour-mass diagram where the galaxies are colour-coded by their local galaxy density. The resulting trend is clear: the very highest and low-mass red galaxies live in the densest regions, while intermediate-mass passive galaxies $\left(M_{*} \sim 10^{10}-10^{11} M_{\odot}\right)$ live in lower-density regions. This trend matches that seen in SDSS observations, at least qualitatively (Hogg et al. 2003; Kauffmann et al. 2004; Blanton et al. 2005). Physically, this trend emerges because massive galaxies with $M_{*} \gtrsim 10^{10.5} M_{\odot}$, once massive enough, can get to the red sequence "on their own" by forming their own hot halos even in (relatively) low-density environments. Massive galaxies that are quenched at early epochs live in the highest overdensities, and thus grow to be the most massive galaxies at $z=0$ by accreting other galaxies (see 4 ). Low-mass $\left(\lesssim 10^{10.5} M_{\odot}\right)$ galaxies can typically only be quenched by living in the hot environment of more massive neighbors.

In the right panel of Figure 6 we show only central galaxies colour-coded by the normalised distance to the nearest halo with a mass $>10^{12} M_{\odot}$. This is roughly the halo mass where hot halos will form independently in our simulations. Gray crosses are for galaxies at the centers of massive halos, and coloured points are for central galaxies in lower-mass halos.

The first clear difference when looking only at centrals is that there is a deficit of $M_{*} \sim 10^{10} M_{\odot}$ red central galaxies, which as we argued in 3.3 arises because red galaxies at that mass are predominantly satellites quenched at late times.

However, there are still some central galaxies that are along the red sequence at intermediate to low masses. Such galaxies at low mass tend to be fairly close to a quenched halo (i.e. the points are blue). This suggests a larger environmental effect - these galaxies are part of super-group structures whose hot gas can be felt beyond the virial radius of the group halo. Thus environmental factors beyond the halo mass may play some role in quenching of star formation. Observations have suggested a role for environment (beyond halo mass) in some galaxy properties (Cooper et al. 2008a, b, 2010), though it is typically secondary to galaxy mass.

Figure 7 shows another representation of the relationship between red fraction and environment. Here we plot contours of red fraction (in colors from blue to red) in the plane of stellar mass versus local galaxy overdensity (determined using Smooth). This figure can be compared to Figure 6 of Peng et al. (2010), who argued for two different quenching mechanisms, namely "environment quenching" of all galaxies above a given overdensity, and "mass quenching" above a given stellar mass.

Our simulation with quenching (left panel) quantitatively reproduces the observed trends almost exactly, although it has far fewer galaxies than in the SDSS data of Peng et al. (2010), and hence the trends are less smooth and there are regions in this space where this simulation yields no galaxies (black). Broadly, our models reproduce the strong increase in red fraction to both high mass and high overdensity, and the "boxy" contour shape noted by Peng et al. $(2010)$. In our simulations, environment quenching is predominantly associated with satellites at lower masses living in hot halos, although as discussed above there are a fair number of small centrals living in dense environments that are also quenched. Meanwhile, mass quenching is well reproduced by our hot gas threshold for quenching, which translates roughly into a halo mass threshold. To a large extent "mass" and "environment" quenching can alternatively be separated into central and satellite quenching (De Lucia et al. 2011b; Peng et al. 2011).

In the right panel of Figure 7 we illustrate "environment quenching" in our simulation without explicit quenching feedback. This simulation produces fewer red galaxies (Gabor et al. 2011), and the figure shows that red galaxies form only at high overdensities. The red galaxies in this case are almost all satellite galaxies. "Mass quenching," indi- 

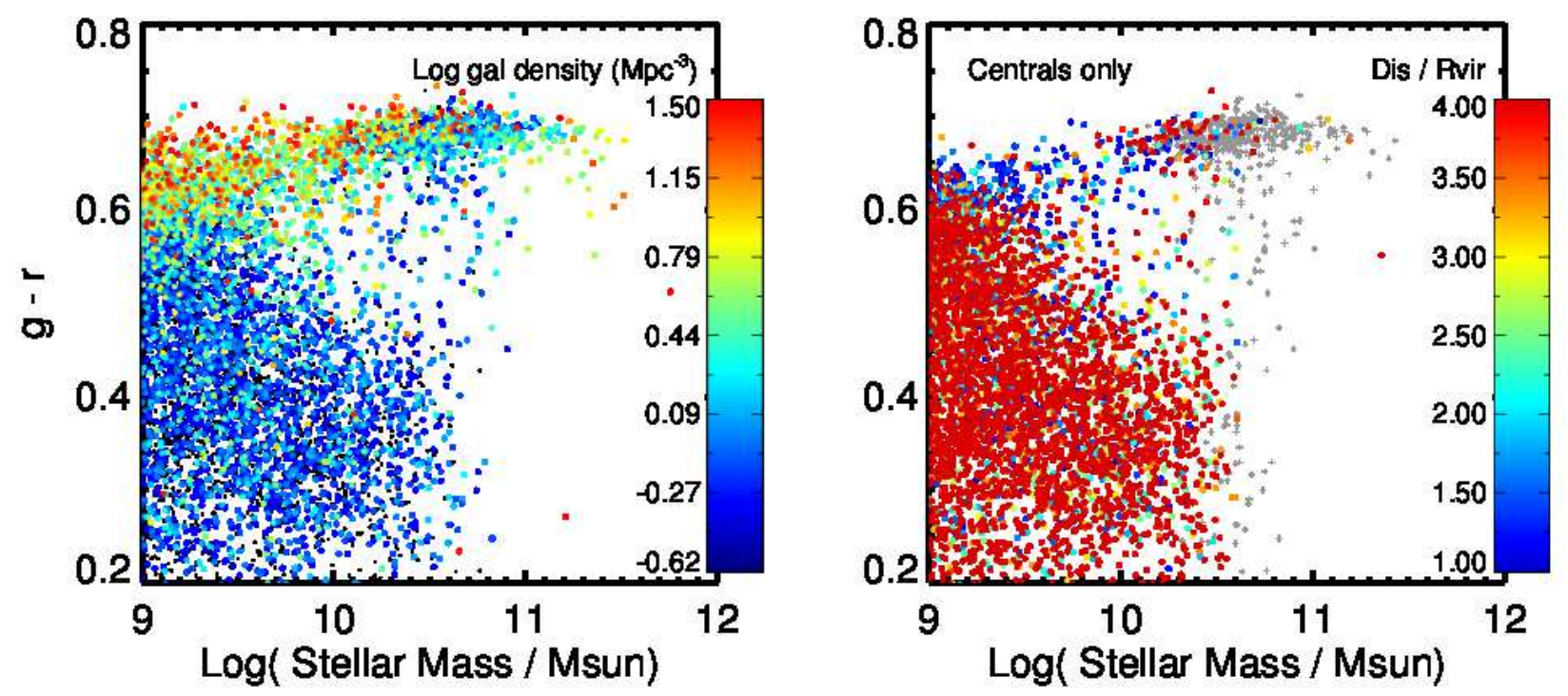

Figure 6. Left CMD (at $z=0$ ) with galaxies colour-coded by the galaxy density within 1 Mpc. The high- and low-mass ends of the red sequence live in dense environments, whereas $\sim L^{*}$ red sequence galaxies tend to live at lower densities. Right Central galaxies are colour-coded by the distance to the closest massive halo (right). Massive halos are defined by a mass of $10^{12} M_{\odot}$, as found using a spherical overdensity algorithm. This is roughly the mass at which a halo is dominated by hot gas (>250,000 K). Grey crosses are galaxies at the centers of their (massive) halos, and blue and red points are outside the virial radius of the nearest massive halo. Some passive galaxies $<10^{10.5} M_{\odot}$ are quenched despite living outside $R_{\mathrm{vir}}$, suggesting the influence of hot gas in unrelaxed super-group environments. Quenched galaxies at $\gtrsim 4 R_{\text {vir }}$ live in halos just below $10^{12} M_{\odot}$, where scatter in the $M_{\text {halo }}$-hot gas relation allows them to be hot-gas-dominated.
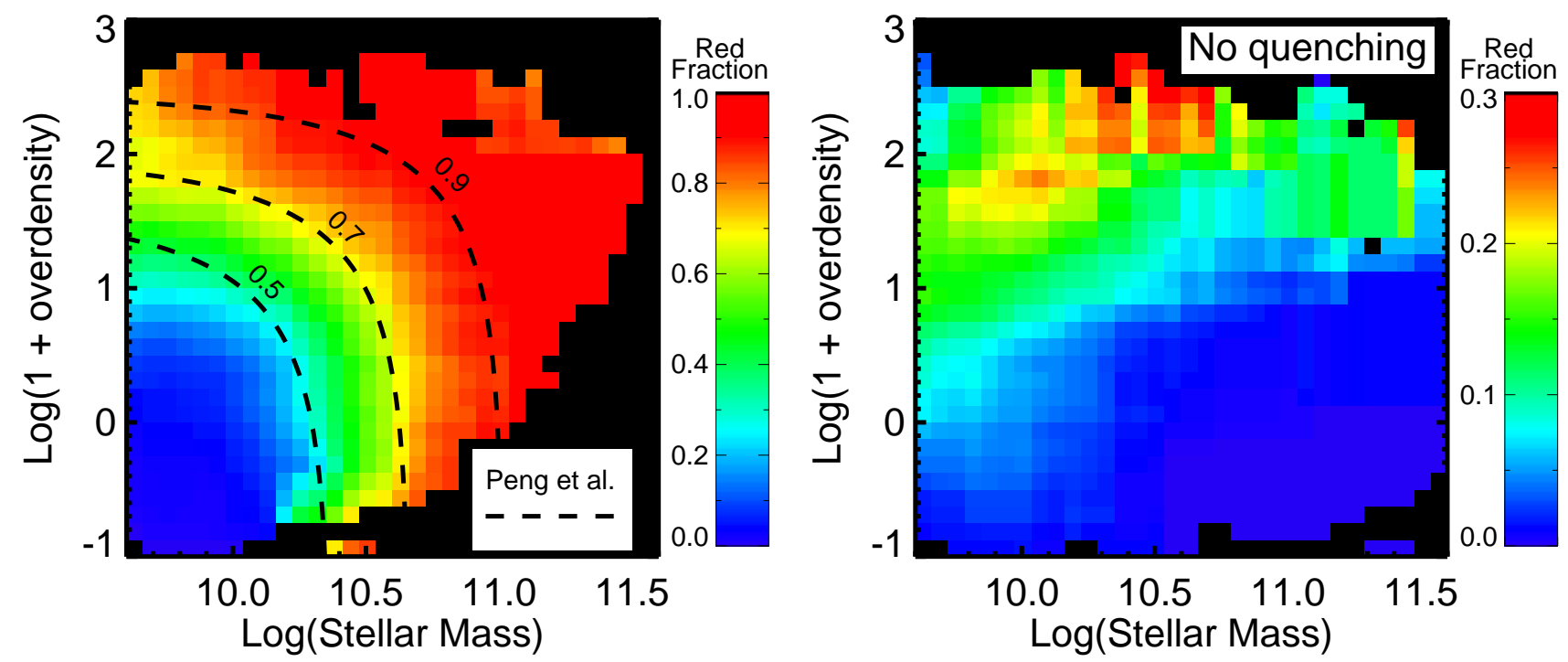

Figure 7. Left: Smoothed distribution of red fraction (colors) in stellar mass vs. overdensity space in our quenching simulation. Galaxies are increasingly red at both higher masses and overdensities. Small galaxies at high overdensity are red because they live within or close to larger halos dominated by hot gas, while larger galaxies are red because they are typically centrals living within hot halos maintained hot via our quenching feedback mechanism. Dashed lines show the best-fit parameterization of red fraction of SDSS data from Peng et al. (2010). Right: Same as left panel, but for a simulation without explicit quenching, and with a different color-scale. Even without adding heat "by hand" in hot halos, some satellite galaxies in high-density regions are quenched. 

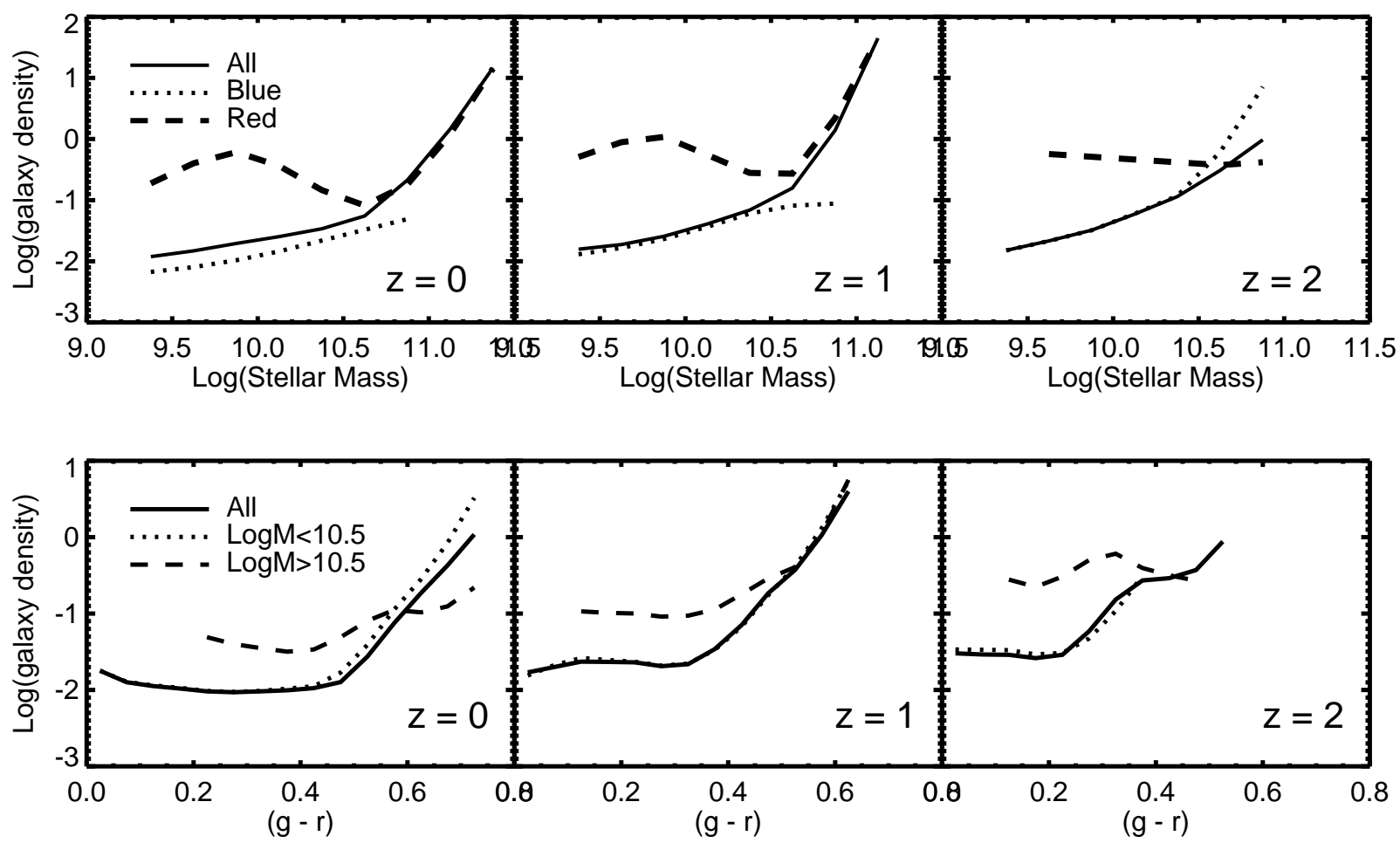

Figure 8. Top row: Mass-density relation for all (solid), blue (dotted), and red (dashed) galaxies at $z=0,1$, and 2 (increasing left-toright). On average (in the "all" sample), galaxies below $10^{10.5} M_{\odot}$ live in low density environments, with a sharp rise in local density at higher masses. Red galaxies at low masses live in high-density environments, producing a pronounced dip in the red galaxy mass-density relation at $\sim 10^{10.5} M_{\odot}$. Redshift evolution to $z=1$ is negligible, and weak at higher $z$. Bottom row: Colour-density relation for all (solid), low-mass (dotted), and high-mass (dashed) galaxies at three redshifts. On average, blue galaxies live in low-density environments and red galaxies in high-density environments. While the overall colour-evolution of galaxies is apparent in the different panels, the basic trends appear roughly the same at all $z$.

cated by vertical contours in the left panel, is a direct result of adding heat to hot halos in our quenching simulation - it is absent from the right panel. Our explicit quenching mechanism also boosts the red fraction among satellites in dense regions.

In summary, our simulations are consistent with the interpretation by Peng et al. (2010) that there are two quenching mechanisms that deliver galaxies to the red sequence, associated with environment and mass, though they can be described more physically as central and satellite quenching. But our simulations further suggest that both mechanisms are ultimately the result of red galaxies living in regions dominated by hot gas, which occurs at both high overdensities and high masses.

\subsection{Evolution of the mass-density and colour-density relations}

We show the evolution of the stellar mass-density relation and the colour-density relation in Figure 8 Here the local galaxy density is found using Smooth.

The mass-density relation (top row of Figure 8) for all galaxies shows a very shallow rise in density with mass for stellar masses below $10^{10.5} M_{\odot}$. Above this mass, density rises steeply. This behavior agrees qualitatively with observations at $z=0$ (Hogg et al. 2003; Blanton et al. 2005). The red galaxy sample departs from the average trend because low-mass red galaxies live in dense regions. For these trends, evolution is negligible out to $z=1$ (top middle panel), but notable at the massive end to $z=2$. This high- $z$ evolution is sensitive to the small numbers of massive galaxies at this redshift, and to the separation between blue and red galaxies, so we do not view it as a strong prediction at this time.

In the colour-density relation (bottom row of Figure 8), density is uniformly low for blue galaxies (low values of $g-r$ ), then rises steeply for red galaxies (higher values of $g-r)$. Massive galaxies $\left(M>10^{10.5} M_{\odot}\right.$, dashed line) live at higher densities with a weaker colour-density trend. Lowmass galaxies, in contrast, must live in dense environments in order to become red. Evolution in the colour-density relation is minor to $z=1$, in accord with recent observations (although there is some debate Cooper et al. 2006, 2007; Elbaz et al. 2007; Scodeggio et al. 2009; Cooper et al. 2010).

In summary, although our quenching model is most directly tied to hot gas fraction and thus halo mass, environment plays a correlated role in quenching galaxies in our simulations. Halo mass is correlated with environment in a CDM universe, so we get the usual mass-density relation. Since our quenching is directly linked to the proportion of hot gas in a halo and thus halo mass (Birnboim \& Dekel 2003; Kereš et al. 2005; Gabor et al. 2011), we also naturally get a pronounced colour-density relation. Evolution in 
the mass-density and colour-density relations is weak in this model and qualitatively consistent with data, perhaps showing an inversion in the colour-density relation only at the highest redshifts considered here.

\section{SUMMARY AND CONCLUSION}

We have analysed a cosmological hydrodynamic simulation to explore the evolution of the red sequence. A key feature of our simulation is a simple quenching prescription tied to the dominance of hot gas within the virial radius of a halo. In our quenching prescription, galaxies whose halos have $>60 \%$ of their baryons in hot gas are starved of star-forming fuel by continual heating of the halo gas. In a previous paper we showed that this simple model yields a distinct red sequence of galaxies whose number densities generally agree with observations (Gabor et al. 2011). This model thus provides a tool for studying the emergence and growth of the red sequence over cosmic time. While some details of our model are overly simplistic and in some cases are in conflict with observations, it serves as representative of a general class of quenching models where cessation of star formation is driven by the presence of hot coronae or is tied to halo mass.

In our simulations, the first galaxies to turn red at $z \gtrsim 2$ are among the most massive in the universe at that epoch, with $M_{\text {stellar }} \sim 10^{10.5-11} M_{\odot}$. Satellite galaxies at low masses are concurrently starved of fuel by the same hot halo that triggers our quenching mechanism, and therefore low mass galaxies also appear on the red sequence at early times. Meanwhile, intermediate-mass galaxies at $\sim 10^{10}-10^{10.5} M_{\odot}$ remain predominantly blue until late epochs, producing a pronounced dip in the red galaxy mass function at high redshifts. This dearth of intermediate-mass red galaxies is qualitatively consistent with observations, but our model may have too many red galaxies with lower masses. At late times, the number of intermediate-mass red galaxies catches up to the number of more massive ones, so that the dip in the red galaxy mass function is less pronounced by $z=0$.

Essentially all simulated passive galaxies with stellar mass $>10^{11} M_{\odot}$ form from smaller passive galaxies that grow mass via mergers once on the red sequence. Most of the mass growth of individual passive galaxies is due to minor mergers, with major mergers being rare and a subdominant component of mass growth. Based on a simple analytic model, these numerous minor mergers can lead to size growth that is a factor of 2 or more times that of a galaxy's corresponding mass growth. This suggests that minor mergers dominate the dramatic size evolution of massive galaxies, in accordance with earlier work Naab et al. 2009; Hopkins et al. 2010a).

The simple quenching model explored here qualitatively reproduces observed trends among colour, stellar mass, and environment at $z=0$. In detail, it also reproduces the observed mass-density trend for the red sequence: low-mass and high-mass passive galaxies live in the densest regions, whereas galaxies in-between (around $M^{*}$ ) live in less dense regions. The mass-density and colour-density trends persist to at least $z=1$. Quenching occurs at both high density, roughly independent of stellar mass, and high stellar mass, roughly independent of density. As argued from correspond- ing observations by Peng et al. (2010), this indicates two quenching processes associated with environment and mass, which in our model both arise due to starvation when the local environment is dominated by hot gas.

Our analysis explictly avoids any considerations of galaxy morphologies. A key question that we therefore do not address is, why are most red and dead galaxies elliptical? While some authors argue that it owes to major mergers that can transform star-forming spirals into elliptical galaxies (Mihos \& Hernquist 1996), the simple cessation of star formation at sufficiently early epochs typically results in substantial later growth via dry (i.e. purely or almost purely stellar) mergers. In this scenario, galaxies would quench first when entering a hot halo (or its immediate environment), become red spirals (Skibba et al. 2009; van der Wel et al. 2009; Bundv et al. 2010; Masters et al. 2010; van der Wel et al. 2011), and then undergo subsequent dry mergers that alter its morphology (e.g. De Lucia \& Blaizot 2007; De Lucia et al. 2011a; Guo \& Oh 2008; Feldmann et al. 2010; Oser et al. 2010, 2012). Our models indicate that larger galaxies living in denser environs undergo more such growth, and so would be expected to have dynamical signatures indicating more dry merging such as boxier isophotes (Naab et al. 2006; Bournaud et al. 2007); these trends are qualitatively consistent with observations. Whether the quantitative predictions of this model are in accord with detailed observations remains to be seen.

More broadly, it appears that a quenching mechanism based on a simple hot gas fraction threshold (which is wellcorrelated with a halo mass threshold) is capable of reproducing a variety of observations not only at the present epoch but also out to $z \sim 1$ and beyond. However, there are significant failures, such as a dearth of the most massive galaxies, and a too-sharp cutoff in the blue galaxy mass function suggesting that both today and at high redshifts in the real Universe, some galaxies are able to be starforming despite living in hot gas-dominated halos. Hence this model represents only a step towards understanding the physical processes driving red galaxy evolution. Comparisons to observations particularly probing the intermediatemass regime out to high redshifts can place strong constraints on models such as these, and highlight additional physical processes that may need to be considered.

\section{ACKNOWLEDGMENTS}

The authors acknowledge K. Finlator, N. Katz, A. Maller, B. D. Oppenheimer, and D. Weinberg for helpful discussions, the referee for expeditious comments, and V. Springel for making Gadget-2 public. The simulations used here were run on the University of Arizona's SGI cluster, ice. This work was supported by the National Science Foundation under grant numbers AST-0847667 and AST-0907998, and by the CANDELS Hubble Multi-Cycle Treasury program. Computing resources were obtained through grant number DMS-0619881 from the National Science Foundation.

\section{REFERENCES}

Abadi M. G., Moore B., Bower R. G., 1999, MNRAS, 308, 
947

Baldry I. K. et al., 2012, MNRAS, 421, 621

Baldry I. K., Glazebrook K., Driver S. P., 2008, MNRAS, 388, 945

Barnes J. E., 1990, Nature, 344, 379

Bekki K., Couch W. J., Shioya Y., 2002, ApJ, 577, 651

Bell E. F. et al., 2004, ApJ, 608, 752

Bezanson R., van Dokkum P. G., Tal T., Marchesini D.,

Kriek M., Franx M., Coppi P., 2009, ApJ, 697, 1290

Binney J., 1977, ApJ, 215, 483

Birnboim Y., Dekel A., 2003, MNRAS, 345, 349

—, 2011, MNRAS, 415, 2566

Birnboim Y., Dekel A., Neistein E., 2007, MNRAS, 380, 339

Blanton M. R., 2006, ApJ, 648, 268

Blanton M. R., Eisenstein D., Hogg D. W., Schlegel D. J., Brinkmann J., 2005, ApJ, 629, 143

Bournaud F., Jog C. J., Combes F., 2007, A\&A, 476, 1179

Bower R. G., Benson A. J., Malbon R., Helly J. C., Frenk C. S., Baugh C. M., Cole S., Lacey C. G., 2006, MNRAS, 370,645

Boylan-Kolchin M., Ma C.-P., Quataert E., 2006, MNRAS, 369, 1081

Brammer G. B. et al., 2011, ApJ, 739, 24

Brammer G. B. et al., 2009, ApJ, 706, L173

Brown M. J. I., Dey A., Jannuzi B. T., Brand K., Benson A. J., Brodwin M., Croton D. J., Eisenhardt P. R., 2007, ApJ, 654, 858

Bruzual G., Charlot S., 2003, MNRAS, 344, 1000

Bundy K. et al., 2010, ApJ, 719, 1969

Cattaneo A., Dekel A., Devriendt J., Guiderdoni B., Blaizot J., 2006, MNRAS, 370, 1651

Cen R., 2011, ApJ, 741, 99

Chabrier G., 2003, ApJ, 586, L133

Cimatti A., Nipoti C., Cassata P., 2012, MNRAS, L424

Cool R. J. et al., 2008, ApJ, 682, 919

Cooper M. C. et al., 2010, MNRAS, 409, 337

Cooper M. C. et al., 2007, MNRAS, 376, 1445

Cooper M. C. et al., 2006, MNRAS, 370, 198

Cooper M. C., Newman J. A., Weiner B. J., Yan R., Willmer C. N. A., Bundy K., Coil A. L., Conselice C. J., Davis M., Faber S. M., Gerke B. F., Guhathakurta P., Koo D. C., Noeske K. G., 2008a, MNRAS, 383, 1058

Cooper M. C., Tremonti C. A., Newman J. A., Zabludoff A. I., 2008b, MNRAS, 390, 245

Cox T. J., Dutta S. N., Di Matteo T., Hernquist L., Hopkins P. F., Robertson B., Springel V., 2006, ApJ, 650, 791

Croton D. J. et al., 2006, MNRAS, 365, 11

Daddi E. et al., 2007, ApJ, 670, 156

Daddi E. et al., 2005, ApJ, 626, 680

Davé R., 2008, MNRAS, 385, 147

Davé R., Finlator K., Oppenheimer B. D., 2006, MNRAS, 370,273

—, 2007, in EAS Publications Series, Vol. 24, EAS Publications Series, E. Emsellem, H. Wozniak, G. Massacrier, J.-F. Gonzalez, J. Devriendt, \& N. Champavert, ed., pp. 183-189

Davé R., Oppenheimer B. D., Finlator K., 2011, MNRAS, 415, 11

De Lucia G., Blaizot J., 2007, MNRAS, 375, 2

De Lucia G., Fontanot F., Wilman D., 2012, MNRAS, 419, 1324
De Lucia G., Fontanot F., Wilman D., Monaco P., 2011a, MNRAS, 414, 1439

De Lucia G., Springel V., White S. D. M., Croton D., Kauffmann G., 2006, MNRAS, 366, 499

De Lucia G., Weinmann S., Poggianti B., AragonSalamanca A., Zaritsky D., 2011b, ArXiv e-prints

Dekel A., Birnboim Y., 2006, MNRAS, 368, 2

一, 2008, MNRAS, 383, 119

Dekel A., Sari R., Ceverino D., 2009, ApJ, 703, 785

Drory N. et al., 2009, ApJ, 707, 1595

Elbaz D. et al., 2007, A\&A, 468, 33

Faber S. M. et al., 2007, ApJ, 665, 265

Fan L., Lapi A., De Zotti G., Danese L., 2008, ApJ, 689, L101

Feldmann R., Carollo C. M., Mayer L., Renzini A., Lake G., Quinn T., Stinson G. S., Yepes G., 2010, ApJ, 709, 218

Finlator K., Davé R., 2008, MNRAS, 385, 2181

Finlator K., Davé R., Oppenheimer B. D., 2007, MNRAS, 376,1861

Finlator K., Davé R., Papovich C., Hernquist L., 2006, ApJ, 639, 672

Gabor J. M., Davé R., Finlator K., Oppenheimer B. D., 2010, MNRAS, 407, 749

—, 2011, MNRAS, 407, 749

Gallagher III J. S., Ostriker J. P., 1972, AJ, 77, 288

Gallazzi A., Charlot S., Brinchmann J., White S. D. M., 2006, MNRAS, 370, 1106

Gao L., White S. D. M., Jenkins A., Stoehr F., Springel V., 2004, MNRAS, 355, 819

Gelb J. M., Bertschinger E., 1994, ApJ, 436, 467

Grogin N. A. et al., 2011, ApJS, 197, 35

Gunn J. E., Gott J. R. I., 1972, ApJ, 176, 1

Guo F., Oh S. P., 2008, MNRAS, 384, 251

Haardt F., Madau P., 2001, in Clusters of Galaxies and the High Redshift Universe Observed in X-rays, D. M. Neumann \& J. T. V. Tran, ed.

Hogg D. W. et al., 2002, AJ, 124, 646

Hogg D. W. et al., 2003, ApJ, 585, L5

Hopkins P. F., Bundy K., Hernquist L., Wuyts S. a nd Cox T. J., 2010a, MNRAS, 401, 1099

Hopkins P. F., Cox T. J., Kereš D., Hernquist L., 2008, ApJS, 175, 390

Hopkins P. F., Croton D., Bundy K., Khochfar S., van den Bosch F., Somerville R. S., Wetzel A., Keres D., Hernquist L., Stewart K., Younger J. D., Genel S., Ma C.-P., 2010b, ApJ, 724, 915

Hopkins P. F., Hernquist L., Cox T. J., Robertson B., Springel V., 2006, ApJS, 163, 50

Jungwiert B., Combes F., Palouš J., 2001, A\&A, 376, 85

Kauffmann G., White S. D. M., Heckman T. M., Ménard B., Brinchmann J., Charlot S., Tremonti C., Brinkmann J., 2004, MNRAS, 353, 713

Kennicutt Jr. R. C., 1998, ApJ, 498, 541

Kereš D., Katz N., Weinberg D. H., Davé R., 2005, MNRAS, 363, 2

Khochfar S., Ostriker J. P., 2008, ApJ, 680, 54

Khochfar S., Silk J., 2006, ApJ, 648, L21

Koekemoer A. M. et al., 2011, ApJS, 197, 36

Komatsu E. et al., 2009, ApJS, 180, 330

Kovač K. et al., 2010, ApJ, 708, 505 
Kriek M., van der Wel A., van Dokkum P. G., Franx M., Illingworth G. D., 2008, ApJ, 682, 896

Larson R. B., Tinsley B. M., Caldwell C. N., 1980, ApJ, 237, 692

Lauer T. R. et al., 2005, AJ, 129, 2138

Maller A. H., Katz N., Kereš D., Davé R., Weinberg D. H., 2006, ApJ, 647, 763

Marchesini D. et al., 2010, ApJ, 725, 1277

Martin C. L., 2005, ApJ, 621, 227

Masters K. L. et al., 2010, MNRAS, 405, 783

Mathews W. G., 2009, ApJ, 695, L49

McKee C. F., Ostriker J. P., 1977, ApJ, 218, 148

Mihos J. C., Hernquist L., 1996, ApJ, 464, 641

Mo H. J., Mao S., White S. D. M., 1998, MNRAS, 295, 319

Moore B., Lake G., Katz N., 1998, ApJ, 495, 139

Mortlock A., Conselice C. J., Bluck A. F. L., Bauer A. E., Grützbauch R., Buitrago F., Ownsworth J., 2011, MNRAS, 413, 2845

Murante G. et al., 2004, ApJ, 607, L83

Murray N., Quataert E., Thompson T. A., 2005, ApJ, 618, 569

Naab T., Johansson P. H., Ostriker J. P., 2009, ApJ, 699, L178

Naab T., Johansson P. H., Ostriker J. P., Efstathiou G., 2007, ApJ, 658, 710

Naab T., Khochfar S., Burkert A., 2006, ApJ, 636, L81

Nipoti C., Treu T., Auger M. W., Bolton A. S., 2009, ApJ, 706, L86

Nipoti C., Treu T., Leauthaud A., Bundy K., Newman A. B., Auger M. W., 2012, ArXiv e-prints

Oppenheimer B. D., Davé R., 2006, MNRAS, 373, 1265

-, 2008, MNRAS, 387, 577

Oppenheimer B. D., Davé R., Kereš D., Fardal M., Katz

N., Kollmeier J. A., Weinberg D. H., 2010, MNRAS, 406, 2325

Oser L., Naab T., Ostriker J. P., Johansson P. H., 2012, ApJ, 744, 63

Oser L., Ostriker J. P., Naab T., Johansson P. H., Burkert A., 2010, ApJ, 725, 2312

Peng Y., Lilly S. J., Renzini A., Carollo M., 2011, ArXiv e-prints

Peng Y.-j. et al., 2010, ApJ, 721, 193

Quilis V., Moore B., Bower R., 2000, Science, 288, 1617

Rees M. J., Ostriker J. P., 1977, MNRAS, 179, 541

Richstone D. O., 1976, ApJ, 204, 642

Rudnick G. et al., 2009, ApJ, 700, 1559

Rudnick G. H., Tran K.-V., Papovich C., Momcheva I., Willmer C., 2012, ArXiv e-prints

Rupke D. S., Veilleux S., Sanders D. B., 2005, ApJS, 160, 115

Salim S. et al., 2007, ApJS, 173, 267

Scannapieco E., Pichon C., Aracil B., Petitjean P., Thacker R. J., Pogosyan D., Bergeron J., Couchman H. M. P., 2006, MNRAS, 365, 615

Scodeggio M. et al., 2009, A\&A, 501, 21

Shankar F., Marulli F., Bernardi M., Dai X., Hyde J. B., Sheth R. K., 2010, MNRAS, 403, 117

Silk J., 1977, ApJ, 211, 638

Simha V., Weinberg D. H., Davé R., Gnedin O. Y., Katz N., Kereš D., 2009, MNRAS, 399, 650

Skibba R. A., 2009, MNRAS, 392, 1467

Skibba R. A. et al., 2009, MNRAS, 399, 966
Somerville R. S., Hopkins P. F., Cox T. J., Robertson B. E., Hernquist L., 2008, MNRAS, 391, 481

Springel V., 2005, MNRAS, 364, 1105

Springel V., Di Matteo T., Hernquist L., 2005, ApJ, 620, L79

Springel V., Hernquist L., 2003, MNRAS, 339, 289

Steidel C. C., Erb D. K., Shapley A. E., Pettini M., Reddy N., Bogosavljević M., Rudie G. C., Rakic O., 2010, ApJ, 717,289

Stutz A. M., Papovich C., Eisenstein D. J., 2008, ApJ, 677, 828

Sutherland R. S., Dopita M. A., 1993, ApJS, 88, 253

Tal T., Wake D. A., van Dokkum P. G., van den Bosch F. C., Schneider D. P., Brinkmann J., Weaver B. A., 2012, ApJ, 746, 138

Taylor E. N. et al., 2009, ApJ, 694, 1171

Toft S. et al., 2007, ApJ, 671, 285

Trager S. C., Faber S. M., Dressler A., 2008, MNRAS, 386, 715

Trager S. C., Worthey G., Faber S. M., Burstein D., Gonzalez J. J., 1998, ApJS, 116, 1

Trujillo I., Conselice C. J., Bundy K., Cooper M. C., Eisenhardt P., Ellis R. S., 2007, MNRAS, 382, 109

Trujillo I., Ferreras I., de La Rosa I. G., 2011, MNRAS, 415, 3903

van der Wel A., Holden B. P., Zirm A. W., Franx M., Rettura A., Illingworth G. D., Ford H. C., 2008, ApJ, 688, 48

van der Wel A., Rix H.-W., Holden B. P., Bell E. F., Robaina A. R., 2009, ApJ, 706, L120

van der Wel A. et al., 2011, ApJ, 730, 38

van Dokkum P. G. et al., 2008, ApJ, 677, L5

van Dokkum P. G. et al., 2010, ApJ, 709, 1018

Wang L., Kauffmann G., 2008, MNRAS, 391, 785

Wetzel A. R., Tinker J. L., Conroy C., 2011, ArXiv e-prints

Whitaker K. E. et al., 2010, ApJ, 719, 1715

White S. D. M., Frenk C. S., 1991, ApJ, 379, 52

White S. D. M., Rees M. J., 1978, MNRAS, 183, 341

Yang X., Mo H. J., van den Bosch F. C., 2009, ApJ, 695, 900 